\title{
Подход к оценке емкости транзитного накопителя
}

\author{
О.И. Kутузов 1, д.m.н., nрофресcop, npoфpeccop, oleg-kutuzov@mail.ru
}

T.M. Татарникова 2, д.m.н., доиент, зав.кафедрой, tm-tatarn@yandex.ru

${ }^{1}$ Санкт-Петербургский государственный электротехнический университет "АЭТИ" им. В.И. Ульянова (Иенина), г. Санкт-Петербург, 197376, Россия

2 Российский государственный гидрометеорологический университет, 2. Санкт-Петербург, 195196, Россия

Определена актуальность задачи оценки емкости накопителя с опасным грузом. Превышение емкости такого накопителя связано с крупными экологическими и/или материальными потерями, поэтому определено как маловероятное событие.

Формально загрузка накопителя представлена как процесс обслуживания поступившей заявки. Для моделирования этого процесса предложено использовать аппарат теории систем массового обслуживания. Показано, что при моделировании процесса обслуживания необходимо учесть неоднородность потока заявок: заявки входного и выходного потоков существенно различаются по объему поступающего в накопитель и извлекаемого из него груза. Во входном потоке заявки поступают пачками, а выходной поток состоит из одиночных заявок.

Предложено использовать аналитико-статистический метод экстремальных значений и модель поведения очереди в буфере с ограниченной емкостью для решения поставленной задачи. Анализ процесса потери заявок при переполнении буфера конечной емкости стационарной системой массового обслуживания сведен к анализу наращиваний и сокращений очереди на интервале регенерации.

Получено выражение для расчетов малых значений вероятностей потерь в буферах конечной емкости в виде аналитико-статистической оценки. Предложена методика сбора и обработки выборочных данных, последовательное выполнение шагов которой позволяет определить необходимую емкость конечного буфера исходя из допустимой вероятности потерь.

Ключевые слова: емкость накопителя, превышение емкости, опасный груз, допустимая вероятность потерь, маловероятное событие, теория систем массового обслужсивания, неоднородный поток заявок, аналитико-статистический метод экстремальных значений.

При транзитных перевозках складирование и промежуточное хранение груза требуют специальных накопителей. Накопитель как всякий динамический объект обладает собственными состояниями в определенные моменты времени. Эти состояния определяются соотношением между интенсивностью поступающего в накопитель потока грузов и интенсивностью потока извлечения грузов из накопителя.

Перевозка опасных грузов, например, химических минеральных удобрений, минеральностроительных грузов, флюсов и т.п., требует специальных накопителей, так как при хранении под открытым небом они не только теряют свое качество, но и экологически опасны.

При перегрузке накопителя такими грузами критичным оказывается некоторое значение емкости накопителя, превышение которого сопряжено с крупными экологическими и/либо материальными потерями. Такое превышение должно быть исключительно маловероятным событием. Возникает задача расчета емкости промежуточного накопителя (буфера) исходя из допустимой вероятности его переполнения, например, из $P_{\text {доп. }} \leq 10^{-9}$.

Формально процесс загрузки накопителя можно представить как обслуживание поступившего требования и использовать для решения поставленной задачи аппарат теории сuстем массового обслуживания (СМО) [1]. Примем условно за $\bar{V}_{\text {вых. }}$ средний объем груза, забираемого из накопителя за один раз. Назовем этот объем пакетом. Пусть $\bar{V}_{\text {вх. }}-$ средний объем груза, доставленный в накопитель. Тогда среднюю наполненность накопителя можно представить в виде дискретной величины:

$$
\lfloor x\rfloor=\left\lfloor\frac{\bar{V}_{\text {вх. }}}{\left.\bar{V}_{\text {вых. }}\right\rfloor,}\right.
$$

где $\lfloor x\rfloor$ - ближайшее целое, не меньшее $x$.

Таким образом, если процесс загрузки накопителя описывать в терминах $\mathrm{CMO}$, то имеем дело с особой неоднородностью потоков: заявки входного и выходного потоков суще- 
ственно различаются по объему поступающего в накопитель и извлекаемого из него груза. Во входном потоке заявки поступают пачками (в каждой пачке $k$ заявок), а выходной поток состоит из одиночных заявок [2].

Теория массового обслуживания располагает точными формулами в явном виде для ограниченного числа схем в мультипликативной форме представления, имеющих дело с однородными потоками [3, 4]. В работе [5] отмечается, что алгоритмический подход должен стать основным в теории СМО.

Для решения поставленной задачи представляется целесообразным использовать аналитико-статистический метод экстремальных значений (extreme values theory - EVT) [6, 7] и модель поведения очереди в буфере с ограниченной емкостью - конечном буфере (КБ).

Модель процесса потерь в буфере конечной емкости. Анализ процесса потери заявок при переполнении КБ стационарной СМО сведен к анализу наращиваний и сокращений очереди на интервале регенерации. В силу стационарности СМО анализ происходящих событий в одном промежутке занятости эквивалентен для всех промежутков. В течение интервала занятости заявки поступают в систему и покидают ее. Если во время занятости обслуживающего прибора в систему поступает более одной заявки (условие 1), то очередь в буфере начинает наращиваться. И, наоборот, очередь сокращается, если за время обслуживания в систему поступает не более одной заявки (условие 2). Сокращение очереди продолжается до тех пор, пока не наступает условие 1 либо не освобождается полностью буфер и кончается промежуток занятости. Количество процессов наращивания и процессов сокращения очереди, происходящих в одном промежутке занятости, одинаково.

Из анализа модели СМО с бесконечнылм буфером (ББ) [8, 9] следует, что потери в СМО с КБ определяются максимальным значением очереди, достигаемым в ББ на одном промежутке занятости независимо от траекторий развития процессов наращивания и сокращения.

И, следовательно, количество теряющихся в КБ заявок из-за переполнения его емкости равно разности между максимальной величиной очереди, образуемой в ББ на промежутке занятости, и значением емкости КБ.

Максимальная величина очереди в ББ, достигаемая в процессе наращивания, не зависит от количества ни процессов наращивания и сокращения, ни заявок, обслуженных в этом про- межутке, то есть не зависит от длины промежутка занятости.

Обозначим $L_{i}$ максимальное значение очереди в ББ, достигнутое на $i$-м промежутке занятости. На значение $L_{i}$ не влияет номер промежутка занятости, оно зависит только от временных интервалов между поступлениями и интервалов обслуживания. Поскольку эти времена являются случайными величинами, $L_{i} \forall i-$ тоже случайная величина. При существовании бесконечного количества промежутков занятости можно утверждать, что максимальная величина очереди $L$ имеет функцию распределения вероятности своих значений.

В итоге это позволяет утверждать, что вероятность потери заявок в КБ емкостью $N$ (вероятность переполнения накопителя) есть функция от хвоста распределения значений максимальной величины $L$, начиная со значения $L=N+1$.

Следовательно, если функция распределения при $L=N$ есть $F(x)$, то вероятность потерь $P_{n}(N)=f[Q(N+1)]=f[1-F(N)]$.

Расчетное выражсение для емкости накопителя (для экспоненциального распределения хвоста). На основе построенной модели и метода EVT получено выражение для расчетов, соответственно, малых $\left(10^{-8}\right.$ и менее) значений вероятностей потерь в буферах конечной емкости - в виде аналитико-статистической оценки

$$
P_{N}=R \frac{a_{n}}{n \bar{K}} e^{-\frac{N-b_{n}+1}{a_{n}}},
$$

где $R$ - нормирующий коэффициент дискретного распределения при использовании метода экстремальных статистик, $R=e^{\Delta x / a n} ; \bar{K}-$ среднее число заявок, поступающих на вход СМО за период регенерации; $n$ - число выборочных значений в последовательностях, на которые разбивается выборочная последовательность, составленная из максимальных значений очереди на интервалах регенерации; $a_{n}$ и $b_{n}-$ коэффициенты, вычисляемые по выборочным данным.

Преобразуем выражение (2), разрешая его относительно требуемого значения $N$, обеспечивающего значение вероятности потери $P(N)$ и не превышающего допустимое значение $P_{\text {доп. }}(N)$. Получаем выражение для оценки емкости промежуточного накопителя:

$$
\lfloor N\rfloor=a_{n} \ln \left[R \frac{a_{n}}{n \bar{K}}-a_{n} \ln P_{\text {доп. }}(N)\right]+b_{n}-1 .
$$

Методика сбора и обработки выборочных данных [10]. Полагаем, что время моделирова- 
ния или объем общей выборки и признак выделения точек регенерации определены на более ранних стадиях моделирования.

Итак, в процессе сбора и обработки данных необходимо выполнить следующее.

1. Выделить интервалы регенерации из общей выборки значений очереди, подсчитать число интервалов и оценить их среднюю длину $\bar{K}$ ( $\bar{K}-$ среднее число заявок, поступивших в систему на интервале регенерации; при поступлении каждой заявки фиксируется значение очереди в системе).

2. По всем интервалам выделить максимальные выборочные значения, которые образуют последовательность независимых значений случайной величины (длины возможной очереди в КБ) для последующей обработки методом EVT.

3. Образовать порядковую последовательность из независимых значений длин очереди и оценить вероятности этих значений.

4. Разделить полученную в п. 2 последовательность на $M$ подпоследовательностей, содержащих по $n$ выборочных значений (значение $n$ можно принять равным 50).

5. Образовать последовательность из максимальных значений $x_{i}, i=\overline{1, M}$, подпоследовательностей.

6. Образовать порядковую последовательность из максимальных значений (п. 5), оценить вероятность каждого значения в ней и построить в виде гистограммы оценку $\bar{H}_{n}(x)$ функции распределения максимального значения выборочной последовательности (п. 2):

$$
\bar{H}_{n}(x)=\frac{\sum_{i} h_{i}}{M+1} ; \quad i=\overline{1, J},
$$

где $h_{i}$ - количество повторов $i$-го выборочного значения.

7. Образовать последовательность значений для каждого $x_{i}$.

8. Оценить значение $a_{n}$ как статистику по формуле

$$
\bar{a}_{n}=\frac{\sum_{i=1}^{J} x_{i} u_{i}-J \bar{x} u}{\sum_{i=1}^{J} u_{i}^{2}-J \bar{u}^{2}},
$$

где $\bar{x}=\frac{1}{J} \sum_{i=1}^{J} x_{i}, \quad \bar{u}=\frac{1}{J} \sum_{i=1}^{J} u_{i}, J-$ число шагов гистограммы, и значение $b_{n}$ по выражению

$$
b_{n}=\bar{x}-\bar{a}_{n} \bar{u} .
$$

9. Исходя из допустимой вероятности потерь определить необходимую емкость $N$ конечного буфера из выражения

$$
\begin{aligned}
& \lceil N\rceil=a_{n} \ln \left[R \frac{a_{n}}{n \bar{K}}-a_{n} \ln P_{\text {доп. }}(N)\right]+b_{n}-1 ; \\
& P_{N}=e^{\Delta x / a_{n}} \frac{a_{n}}{n \bar{K}} e^{-\left(N-b_{n}+1\right) / a_{n}},
\end{aligned}
$$

где $R=e^{\Delta x / a_{n}}, 2 \Delta x=h_{i}-h_{i-1}-$ длительность интервала между значениями $h_{i}, h_{i-1}$ дискретной величины.

В таблице представлены результаты расчетов, выполненных по формуле (7). При расчетах использованы значения выборочных статистик $\bar{K}, n, R, a_{n}$ и $b_{n}$. Для каждого значения $\rho$ получены параметры выражения (7), вычисляемые по выборочным данным:

- $\rho=0,5 ; n=50 ; n \bar{K}=230 ; R=1,575$; $a_{n}=1,1 ; b_{n}=3,3$.

- $\rho=0,7 ; n=50 ; n \bar{K}=310 ; R=1,5$; $a_{n}=1,3 ; b_{n}=4,7$.

- $\rho=0,9 ; n=50 ; n \bar{K}=890 ; R=1,1$; $a_{n}=4,3 ; b_{n}=12$.

Значения емкости конечного буфера при допустимой вероятности потерь

The values of the final buffer capacity with a permissible probability of loss

\begin{tabular}{|c|c|c|c|}
\hline \multirow{2}{*}{$\boldsymbol{P}_{\text {доп. }}$} & \multicolumn{3}{|c|}{$\boldsymbol{\rho}$} \\
\cline { 2 - 4 } & $\mathbf{0 , 5}$ & $\mathbf{0 , 7}$ & $\mathbf{0 , 9}$ \\
\hline $10^{-5}$ & 14 & 23 & 82 \\
\hline $10^{-7}$ & 21 & 34 & 123 \\
\hline $10^{-8}$ & 25 & 39 & 144 \\
\hline $10^{-9}$ & 28 & 44 & 165 \\
\hline $10^{-11}$ & 35 & 55 & 206 \\
\hline
\end{tabular}

Данные таблицы соответствуют физике явления: во-первых, чем меньше значение допустимой вероятности потерь заявок входного потока, тем больше должна быть емкость конечного буфера; во-вторых, с возрастанием нагрузки увеличивается значение требуемой емкости конечного буфера, обеспечивающего допустимую вероятность потерь.

Заключение. При моделировании процесса обслуживания необходимо учесть неоднородность потока заявок: заявки входного и выходного потоков существенно различаются по объему поступающего в накопитель и извлекаемого из него груза. Во входном потоке заявки поступают пачками, а выходной поток состоит из одиночных заявок.

Предложено использовать аналитико-статистический метод экстремальных значений и модель поведения очереди в буфере с ограниченной емкостью для решения поставленной задачи. Анализ процесса потери заявок при пе- 
реполнении буфера конечной емкости стационарной системой массового обслуживания сведен к анализу наращиваний и сокращений очереди на интервале регенерации. Получено выражение для расчетов малых значений вероятностей потерь в буферах конечной емко- сти в виде аналитико-статистической оценки. Предложена методика сбора и обработки выборочных данных, последовательное выполнение шагов которой позволяет определить необходимую емкость КБ исходя из допустимой вероятности потерь.

\section{Лuтература}

1. Карсаев О.В., Кулемин В.Ю., Морозов Б.М. Планирование доставки сборных грузов. Тр. СПИИРАН. 2014. № 6. С. 5-20.

2. Зуева О.Н., Сидоренко А.М., Галактионов А.Д. Имитационное моделирование доставки грузов с помощью сменных кузовов // Управленец. 2017. № 6. С. 80-86.

3. Бояршинова И.Н., Исмагилов Т.Р., Потапова И.А. Моделирование и оптимизация работы системы массового обслуживания // Фундаментальные исследования. 2015. № 9-1. С. 9-13.

4. Задорожный В.Н. Оптимизация маршрутных матриц в сетях с очередями // Омский науч. вестн. 2016. № 6. С. 152-157.

5. Рыжиков Ю.И. Алгоритмический подход к задачам массового обслуживания. СПб: Изд-во ВКА им. А.Ф. Можайского, 2013. 496 с.

6. Королев В.Ю., Соколов И.А. Об условиях сходимости распределений экстремальных порядковых статистик к распределению Вейбулла // Информатика и ее применение. 2014. № 3. С. 3-11.

7. Быков А.А. Приложения асимптотической теории вероятностей экстремальных значений к прогнозированию риска экстремальных чрезвычайных ситуаций // Проблемы прогнозирования. 2012. № 1. C. 53-64.

8. Kutuzov O., Tatarnikova T. Evaluation and comparison of classical and fractal queuing systems. Proc. 15th Int. Sympos. Redundancy, 2016, pp. 155-157. DOI: 10.1109/RED.2016.7779352.

9. Kutuzov O.I., Tatarnikova T.M. Model of a self-similar traffic generator and evaluation of buffer storage for classical and fractal queuing system. Proc. WS MWENT, 2018, pp. 1-3. DOI: 10.1109/mwent.2018. 8337306.

10. Bernabei F., Ferretti R., Listanti M., Zingrillo G. A tethodology for buffer design in ATM switches. J. European Transactions on Telecommunications and Related Technologies. 1991, vol. 2, no. 4, pp. 367-379.

\section{The assessment approach to the transit drive holding capacity}

O.I. Kutuzov ${ }^{1}$, Dr.Sc. (Engineering), Professor, Professor, oleg-kutuzov@mail.ru

T.M. Tatarnikova ${ }^{2}$, Dr.Sc. (Engineering), Associate Professor, Head of Chair, tm-tatarn@yandex.ru

${ }^{1}$ St. Petersburg Electrotechnical University "LETI", St. Petersburg, 197376, Russian Federation

${ }^{2}$ Russian State Hydrometeorological University, St. Petersburg, 195196, Russian Federation

Abstract. This paper appoints the problem actuality of the holding capacity assessment with dangerous freight. The holding capacity transcendence of such hoarder is connected with the large environmental or financial loss; accordingly, it is a remote contingency.

Legally the drive loading is a service operation of the received application. The machinery of the theory congestion system is for modeling of the received application process. The authors showed that it is necessary to take into account the stream no uniformity of orders in service operation modeling. The input and output stream orders are vary wildly in the extent of incoming and out coming freight from it. In the input stream, the orders come by batches, but the output stream has unicast orders.

The authors offer to use the analytico-statistical method of the extreme values and the behavioral model of the sequence in the buffer with the limited capacity for solving the original problem. The analysis of the order losing process in the overcrowded buffer of the terminal capacity by the stationary system of mass service is reduced to the analysis of the sequence build-up and build-down on the refresh interval. 
The authors got the expression for the low value calculation of the discard probability in the terminal capacity buffer in the form of the analytico-statistical value. The authors offered the acquisition and computing technique of the sampled data. Performing sequential steps it makes possible to estimate the required capacity of the final buffer according to the waste tolerance probability.

Keywords: storage capacity, over capacity, dangerous freight, waste tolerance probability, remote contingency, congestion system theory, nonuniform stream orders, analytico-statistical method of the extreme values.

\section{References}

1. Karsaev O.V., Kulemin V.Yu., Morozov B.M. Assorted cargo delivery scheduling. Proc. of SPIIRAS. 2014, vol. 37, no. 6, pp. 5-20 (in Russ.).

2. Zueva O.N., Sidorenko A.M., Galaktionov A.D. Multi-agent simulation for freight transportation with swap bodies. The Manager. 2017, vol. 70, no. 6, pp. 80-86 (in Russ.).

3. Boyarshinova I.N., Ismagilov T.R., Potapova I.A. Simulation and optimization of queuing systems. Fundamental Research. 2015, no. 9-1, pp. 9-13 (in Russ.).

4. Zadorozhny V.N. Optimization of transition probabilities in networks with queues. The J. Omsk Sci. Bull. 2016, no. 6, pp. 152-157 (in Russ.).

5. Ryzhikov Yu.I. Algorithmic Approach to Queuing Problems. St. Petersburg, 2013, 496 p.

6. Korolev V.Yu., Sokolov I.A. On conditions of convergence of the distributions of extremal order statistics to the Weibull distribution. Informatics and Its Application. 2014, vol. 8, no. 3, pp. 3-11 (in Russ.).

7. Bykov A.A. Applications of the asymptotic theory of probabilities of extreme values to forecasting the risk of extreme emergencies. Forecasting Problems. 2012, vol. 2, no. 1, pp. 53-64 (in Russ.).

8. Kutuzov O., Tatarnikova T. Evaluation and comparison of classical and fractal queuing systems. Proc. 15th Int. Sympos. Redundancy. 2016, pp. 155-157. DOI: 10.1109/RED.2016.7779352.

9. Kutuzov O.I., Tatarnikova T.M. Model of a self-similar traffic generator and evaluation of buffer storage for classical and fractal queuing system. Proc. WS MWENT. 2018, pp. 1-3. DOI: 10.1109/mwent.2018. 8337306.

10. Bernabei F., Ferretti R., Listanti M., Zingrillo G. A tethodology for buffer design in ATM switches. J. Europ. Trans. on Telecommunications and Related Technologies. 1991, vol. 2, no. 4, pp. 367-379.

\section{Для цитирования}

Кутузов О.И., Татарникова Т.М. Подход к оценке емкости транзитного накопителя // Программные продукты и системы. 2020. Т. 33. № 1. С. 033-037. DOI: 10.15827/0236-235X.129. 033-037.

\section{For citation}

Kutuzov O.I., Tatarnikova T.M. The assessment approach to the transit drive holding capacity. Software \& Systems. 2020, vol. 33, no. 1, pp. 033-037 (in Russ.). DOI: 10.15827/0236-235X.129. 033-037. 